\title{
Electrical Properties of the Thin Films Using a Low Temperature Supercritical Carbon Dioxide Fluid Process
}

\author{
Kai-Huang Chen, Sean Wu, and Chien-Min Cheng
}

\begin{abstract}
Electrical and physical properties of as-deposited BLTV ferroelectric thin films on $\mathrm{SiO}_{2} / \mathrm{Si}(100)$ substrates were improved by low temperature supercritical carbon dioxide fluid (SCF) process treatment. The as-deposited BLTV ferroelectric thin films were treated by SCF process which mixed with propyl alcohol and pure water. The memory windows increased in $\mathrm{C}-\mathrm{V}$ curves, and the oxygen vacancy and defect in leakage current density curves were obtained after SCF process treatment. Finally, the improvement properties of as-deposited BLTV thin films after SCF process treatment were investigated by XPS, C-V, and J-E measurement. The mechanism concerning the dependence of electrical properties of the ferroelectric thin films on the SCF process was investigated and discussed.
\end{abstract}

Index Terms-SCF process, BLTV thin film, memory window, leakage current density, NvFeRAM.

\section{INTRODUCTION}

Many ferroelectric materials, such as pervoskite $\left(\mathrm{ABO}_{3}\right)$ and Bi-layer ferroelectrics (BLFS) had been widely investigated for the applications in non-volatile ferroelectric random access memory devices in recently year. The conventional ferroelectric materials such as $\mathrm{Pb}(\mathrm{ZrTi}) \mathrm{O}_{3}$ (PZT), $\mathrm{SrBi}_{2} \mathrm{Ta}_{2} \mathrm{O}_{9}(\mathrm{SBT}), \mathrm{SrTiO}_{3}$ and $(\mathrm{Ba}, \mathrm{Sr}) \mathrm{TiO}_{3}(\mathrm{BST})$ have been widely investigated and developed for the application dynamic random access memory with large storage capacity and non-destructive read out mode non-volatile memory devices [1]-[9].

Recently, electronic devices and systems on panel (SOP) technology had been widely discussed and researched. However, the high-temperature fabrication process for electronic devices is sometimes essential and indispensable technology, such as the conventional temperature annealing (CTA), rapid thermal annealing (RTA) etc. The indium-tin-oxide (ITO) glass substrate would be deformed and fused under high temperature process. To improve the characteristics of thin films, it is necessary to decrease the processing temperature and increase the compatibility of the fabrication of thin films with the integrated circuit processing.

Low temperature fabrication process for thin films

Manuscript received November 22, 2014; revised March 15, 2015. This work was supported in National Science Council of the Republic of China (NSC 100-2221-E-272-003).

Kai-Huang Chen and Sean-Wu were with the Department of Electronics Engineering and Computer Science, Tung-Fang Design Institute Kaohsiung, R.O.C., Taiwan (e-mail: d9131802@gmail.com, wu.sean@gmail.com).

Chien-Min Cheng was with the Department of Electronic Engineering, Southern Taiwan University of Science and Technology, Tainan, R.O.C., Taiwan (e-mail: ccmin523@gmail.com). crystallization and quality for electronic devices was essential and indispensable technology. To improve the physical and electrical properties of semiconductor thin films, the low processing temperature and the compatibility of the integrated circuit fabrication and processing was necessary. The capability properties of the liquid-like supercritical $\mathrm{CO}_{2}$ $\left(\mathrm{SCCO}_{2}\right)$ fluid process was attracted considerable research in transported the $\mathrm{H}_{2} \mathrm{O}$ molecules efficiently and diffusion into the thin films at a low temperature treatment [1]-[3]. The improvement in phenomena and performance of the $\mathrm{SCCO}_{2}$ fluid technology at low temperature were investigated to terminate traps in thin film [4]-[15].

Ferroelectric thin films were focused on the applications in ferroelectric random access memory (FeRAMs), such as portable electrical devices and smart cards utilizing large remnant polarization (2Pr), low coercive field, fatigue-free, low electric consumption, and non-volatility. In pervious study, the BIT materials exhibit high leakage current and domain pinning properties because of the defects such as bismuth and oxygen vacancies. The BTV thin film was prepared by substituting a bismuth ion with a lanthanum ion at A-site substitution, and the fatigue endurance characteristics was improved

In this study, the electrical and physical properties of the as-deposited BLTV thin films prepared on $\mathrm{Pt} / \mathrm{TiO}_{2} / \mathrm{SiO}_{2} / \mathrm{Si}(100)$ substrate were investigated. The phenomena improvements in performance of the SCF process technology at low temperatures were discussed to terminate traps in as-deposited BLTV thin films.

\section{EXPERIMENTAL DETAILS}

The $\left(\mathrm{Bi}_{3.9} \mathrm{La}_{0.1}\right)\left(\mathrm{Ti}_{0.9} \mathrm{~V}_{0.1}\right) \mathrm{O}_{3}$ (BLTV) composition of ceramic target prepared, the $\mathrm{Bi}_{2} \mathrm{O}_{3}, \mathrm{La}_{2} \mathrm{O}_{3}, \mathrm{~V}_{2} \mathrm{O}_{5}$, and $\mathrm{TiO}_{2}$, raw materials were mixed and fabricated by solid state reaction method. After mixing and ball-milling, the mixture was dried, grounded, and calcined at $800^{\circ} \mathrm{C}$ for $2 \mathrm{~h}$. Then, the pressed BLTV target with a diameter of two inches was sintered at $1100^{\circ} \mathrm{C}$ in ambient air for $2 \mathrm{~h}$. The metal-ferroelectric-semiconductor (MFM) structures were shown in Fig. 1. To passivate the traps in BLTV thin films placed in a supercritical fluid system at $150{ }^{\circ} \mathrm{C}$ for 1 hour, it was injected with 3000 psi $\mathrm{SCCO}_{2}$ fluids which were mixed with 10 vol \% pure $\mathrm{H} 2 \mathrm{O}$ and 10 vol \% propyl alcohol. The propyl alcohol was act the role of surfactant between the $\mathrm{SCCO}_{2}$ fluids and polar- $\mathrm{H}_{2} \mathrm{O}$ molecules. The crystal structures of the as-deposited BLTV thin films were determined by X-ray diffraction analysis obtained using a $\mathrm{Cu}$ $\mathrm{K} \alpha$ radiation in the $2 \theta$ range of $20^{\circ}-60^{\circ}$.

The chemical bonding state and oxygen content of BLTV 
thin films were detected by x-ray photoelectron spectroscopy (XPS). The surface micro structure was observed using a scanning electron microscopy. The dielectric constants and leakage current characteristics of BLTV thin films were measured using a gain phase analyzer (HP 4194A) and a semiconductor parameter analyzer (HP 4156). All the capacitance voltage results were measured at $100 \mathrm{kHz}$, with the initial dc bias at the top electrode scanned between -20 and 20 volts.

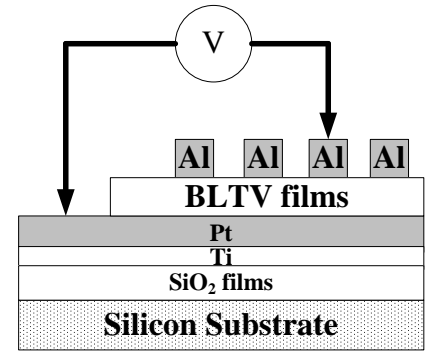

Fig. 1. The metal-ferroelectric- metal (MFM) capacitor structure.

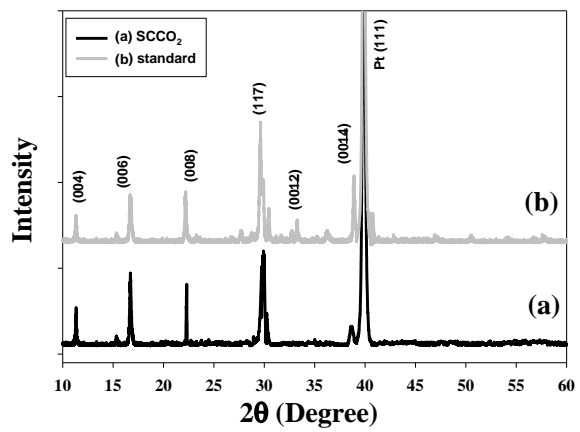

Fig. 2. The XRD patterns of as-deposited BLTV thin films treated by $\mathrm{SCCO}_{2}$ treatment.

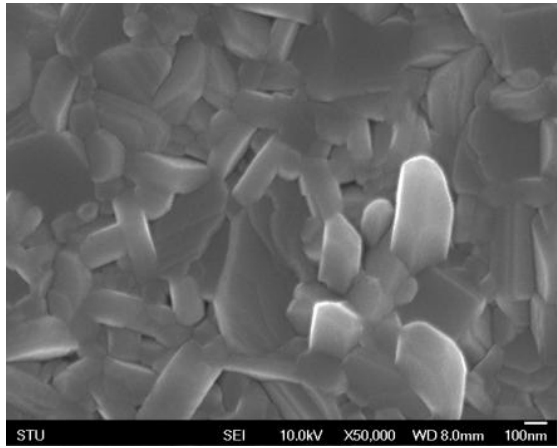

Fig. 3. The surface observation of the as-deposited BLTV thin films.

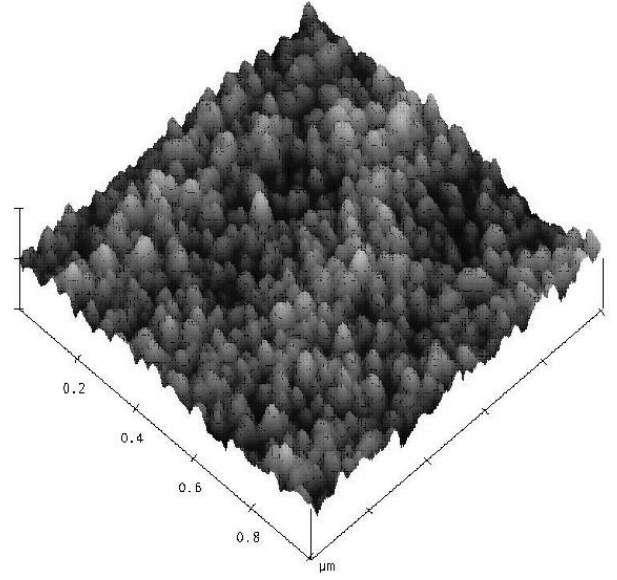

Fig. 4. The AFM morphology of the as-deposited BLTV thin films.

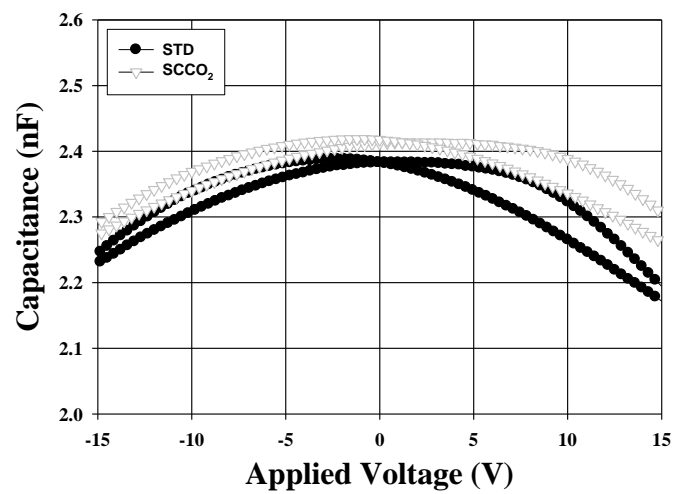

Fig. 5. The capacitance versus applied voltage $(\mathrm{C}-\mathrm{V})$ characteristics of BLTV thin films using SCCO2 treatment.

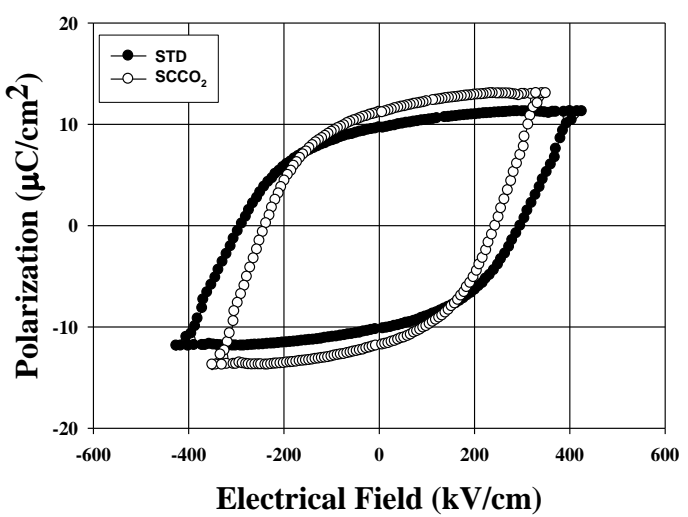

Fig. 6. The polarization versus electrical field (p-E) characteristics of BLTV thin films using SCCO2 treatment.

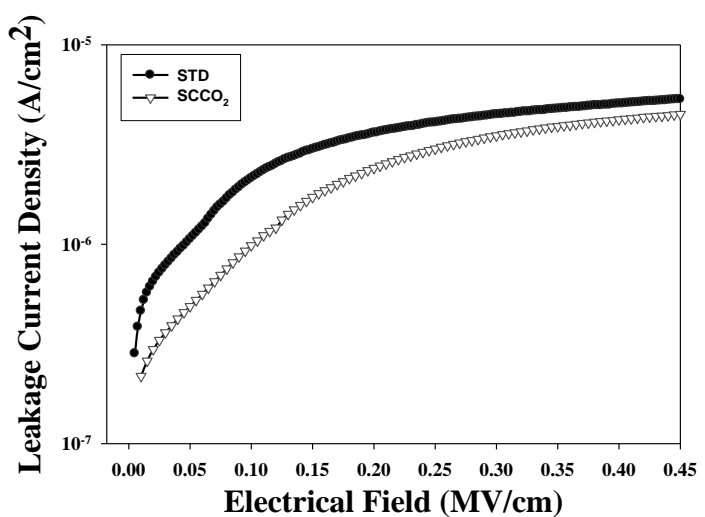

Fig. 7. The leakage current density versus electrical field (J-E) characteristics of BLTV thin films using $\mathrm{SCCO}_{2}$ treatment.

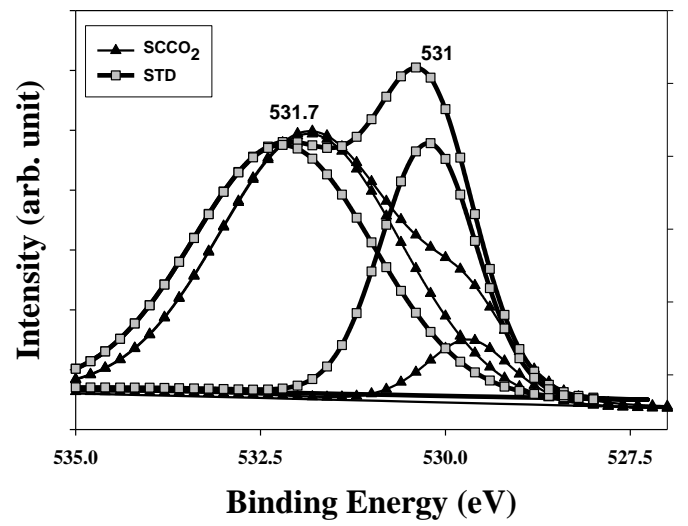

Fig. 8. XPS spectra of O 1s energy levels of as-deposited BLTV thin films after SCF treatment. 


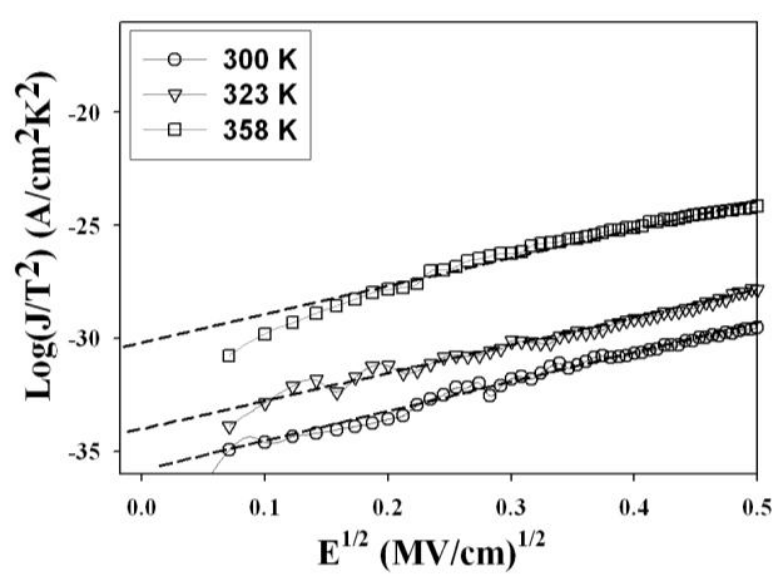

Fig. 9. The leakage current density versus electrical field characteristics in terms of $\mathrm{J} / \mathrm{T}^{2}$ as vertical axis and $\mathrm{E}^{1 / 2}$ as horizontal axis.

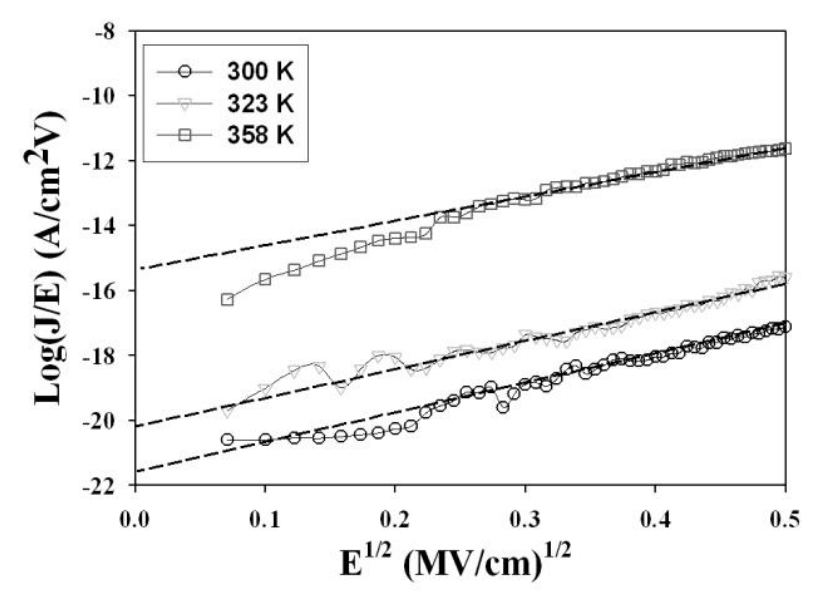

Fig. 10. The same $J-E$ characteristics in terms of $J / E$ as vertical axis and $E^{1 / 2}$ as horizontal axis.

\section{RESULTS AND DISCUSSION}

Fig. 2 shows the X-ray diffraction pattern of BLTV thin film on $\mathrm{SiO}_{2} / \mathrm{Si}$ substrate by rf sputtering technology. From the XRD results, the as-deposited BLTV thin films were polycrystalline structure. The (004), (006), (118), and (117) peaks of as-deposited thin film were found. This result indicated that the crystalline characteristics of BLTV thin films treated by $\mathrm{SCCO}_{2}$ method were not changed for thin films deposited at room temperature. In addition, we found that the (006) and (117) peaks of the $\mathrm{SCCO}_{2}$ fluid treatment films and non-treatment BLTV thin films were also not changed.

The epitaxially as-deposited BLTV thin films on $\mathrm{Pt} / \mathrm{TiO}_{2} / \mathrm{SiO}_{2} / \mathrm{Si}$ substrate were shown in Fig. 3. From the surface morphology, circular-like grains with $200 \mathrm{~nm}$ were observed with scanning electron microscopy for as-deposited BLTV thin films. The as-deposited BLTV thin film thicknesses were measured to be $300 \mathrm{~nm}$ from the cross-sectional morphology. Besides, the surface roughness of as-deposited BLTV thin films formed under the optimal deposition parameters were determined by AFM. The grain size and roughness of as-deposited BLTV thin films were calculated using the images in Fig. 4. The roughness of as-deposited BLTV thin films was 5.742. From the results obtained, the surface roughness and nuclear reaction rate of as-deposition BLTV thin films were attributed to the deposited substrate temperature.

Fig. 5 shows the different in the capacitance versus the applied voltage $(\mathrm{C}-\mathrm{V})$ of the $\mathrm{SCCO}_{2}$ treated and non-treated BLTV thin films measured at $100 \mathrm{kHz}$. The applied voltages, which were first changed from -15 to $15 \mathrm{~V}$ and then returned to $-15 \mathrm{~V}$, were used to measure the capacitance voltage characteristics $(\mathrm{C}-\mathrm{V})$. The $\mathrm{SCCO}_{2}$ treated BLTV thin films exhibited high capacitance than those of non-treated thin films. We found that the capacitances of the $\mathrm{SCCO}_{2}$ treated BLTV thin films were increased from 2.4 to $2.42 \mathrm{nF}$.

Fig. 6 shows the p-E curves of the $\mathrm{SCCO}_{2}$ treated and non-treated BLTV thin films under applied voltage of $20 \mathrm{~V}$ from the Sawyer Tower circuits. The remanent polarization of the $\mathrm{SCCO}_{2}$ treated and non-treated BLTV thin films linearly were increased from 9.5 to $11 \mu \mathrm{C} / \mathrm{cm}^{2}$, respectively. The coercive filed of non-doped, vanadium-doped, and lanthanum-doped ferroelectric thin films were about 300 and $250 \mathrm{kV} / \mathrm{cm}$, respectively. The ferroelectric properties of $\mathrm{SCCO}_{2}$ treated and BLTV thin films were improved and observed.

Fig. 7 shows the leakage current density versus electrical field (J-E) characteristics of the $\mathrm{SCCO}_{2}$ treated and non-treated BLTV thin films. The leakage current density of the $\mathrm{SCCO}_{2}$ treated thin films were about one order of magnitude lower than those of the non-treated BLTV thin films. We suggested that low leakage current density attributed to oxygen atom into vacancy of the $\mathrm{SCCO}_{2}$ treated thin films. To discuss oxygen atom into vacancy of thin films, the leakage current versus electrical field curves of BLTV thin films were fitted to Schottky emission and Poole-Frankel transport models.

To investigate the variation in chemical bonding of the $\mathrm{SCCO}_{2}$ treated and non-treated BLTV thin films prepared by SCF post-treatment process, the doublet structure was observed in the XPS spectrum of $\mathrm{O} 1 \mathrm{~s}$ peak were shown in Fig. 8. Its component peak was fitted to low binding energy and high binding energy peaks at 529 and $531 \mathrm{eV}$, respectively. In Fig. 8, these results reveled that the $\mathrm{H}_{2} \mathrm{O}$ molecules were operatively react in dangling bonds and traps of the $\mathrm{SCCO}_{2}$ treated thin films after SCF treatment. The strong $\mathrm{O}$ 1s bonding of the as-deposited thin film after SCF treatment was observed [16]-[25].

Fig. 9 shows the leakage current density versus electrical field characteristics in terms of $\mathrm{J} / \mathrm{T}^{2}$ as vertical axis and $\mathrm{E}^{1 / 2}$ as horizontal axis. If the J-E curves obey the schottky emission model, the fitting curves should be straight in this figure. For $\mathrm{SCCO}_{2}$ fluid treatment, the current of thin films was fitted well by straight lines in this study.

Fig. 10 also showed the same J-E characteristics in terms of $J / E$ as vertical axis and $E^{1 / 2}$ as horizontal axis. The fitting curves were straight in this figure and J-E curves of thin films should be the Poole-Frankel emission model. From above results, the leakage current of thin films after the $\mathrm{SCCO}_{2}$ fluid treatment process were fitted well by straight lines above the electrical field of $250 \mathrm{kV} / \mathrm{cm}$. Therefore, these results suggested that oxygen vacancies of thin films after the $\mathrm{SCCO}_{2}$ fluid treatment process would be decreased. We induced that the higher leakage current density for films attributed to the more oxygen defect and vacancies. 
In conclusion, the $\mathrm{SCCO}_{2}$ fluid technology was an effective method to remove the charges and defects for ferroelectric thin films. The $\mathrm{SCCO}_{2}$ fluid treatment was developed to take the $\mathrm{H}_{2} \mathrm{O}$ molecules terminate the traps and oxidization with $\mathrm{H}_{2} \mathrm{O}$ molecules for $\mathrm{Ba}\left(\mathrm{Zr}_{0.1} \mathrm{Ti}_{0.9}\right) \mathrm{O}_{3}$ thin films. In addition, the maximum capacitance and lower leakage current density were determined to be $3 \mathrm{nF}$ and $10^{-7}$ $\mathrm{MV} / \mathrm{cm}$, respectively. The improvement in the remnant polarization of the $\mathrm{Ba}\left(\mathrm{Zr}_{0.1} \mathrm{Ti}_{0.9}\right) \mathrm{O}_{3}$ thin film using $\mathrm{SCCO}_{2}$ fluid treatment were also observed. Therefore, the effective dielectric constant increased, reduction of interface states and passivation of traps of the $\mathrm{Ba}\left(\mathrm{Zr}_{0.1} \mathrm{Ti}_{0.9}\right) \mathrm{O}_{3}$ thin films for $\mathrm{SCCO}_{2}$ fluid treatment would be expected to play an important role for the applications in nonvolatile memory devices.

\section{CONCLUSION}

In this study, the SCF technology was an effective method to improve the oxygen vacancy and defects for as-deposited semiconductor ferroelectric thin films. The high capacitance and low leakage current density were determined. They were about $2.42 \mathrm{nF}$ and $10^{-6} \mathrm{~A} / \mathrm{cm}^{2}$, respectively. The improvements in the electrical properties of the as-deposited ferroelectric thin films using SCF treatment were also observed. Therefore, the coercive filed for reduction of oxygen vacancy and passivated of defects in the as-deposited thin films using SCF treatment were observed. The low temperature SCF post-treatment process was an important role for the applications in nonvolatile ferroelectric memory devices.

\section{ACKNOWLEDGEMENTS}

This work will acknowledge the financial support of the National Science Council of the Republic of China (NSC 100-2221-E-272-003).

\section{REFERENCES}

[1] P. C. Yang, T. C. Chang, S. C. Chen, Y. S. Lin, H. C. Huang, and D. S. Gan, "Influence of bias-induced copper diffusion on the resistive switching characteristics of SiON thin film," Electrochem. Solid State Lett., vol. 14, no. 2, 93-95, Oct. 2011.

[2] Y. E. Syu, T. C. Chang, T. M. Tsai, Y. C. Hung, K. C. Chang, M. J. Tsai, M. J. Kao, and S. M. Sze, "Redox reaction switching mechanism in RRAM device with $\mathrm{Pt} / \mathrm{CoSiOX} / \mathrm{TiN}$ structure", IEEE Electron Device Lett., vol. 32, no. 4, pp. 545-547, April, 2011.

[3] L. W. Feng, C. Y. Chang, Y. F. Chang, W. R. Chen, S. Y. Wang, P. W. Chiang, and T. C. Chang, "A study of resistive switching effects on a thin $\mathrm{FeOx}$ transition layer produced at the oxide/iron interface of TiN/SiO2/Fe-contented electrode structures," Appl. Phys. Lett. vol. 96, p. 52111 , Feb. 2010.

[4] L. W. Feng, C. Y. Chang, Y. F. Chang, T. C. Chang, S. Y. Wang, S. C. Chen, C. C. Lin, S. C. Chen, and P. W. Chiang, "Improvement of resistance switching characteristics in a thin $\mathrm{FeOx}$ transition layer of TiN/SiO2/FeOx/FePt structure by rapid annealing," Appl. Phys. Lett. vol. 96 , p. 222108, Jun. 2010.

[5] M. C. Chen, T. C. Chang, C. T. Tsai, S. Y. Huang, S. C. Chen, C. W. Hu S. M. Sze, and M. J. Tsai, "Influence of electrode material on the resistive memory switching property of indium gallium zinc oxide thin films," Appl. Phys. Lett. vol. 96, p. 262110, Jun. 2010.

[6] L. Qi, L. Shibing, W. Wei, Z. Qingyun, Z. Sen, C. Junning, and L. Ming, "Improvement of resistive switching properties in ZrO2-based ReRAM with implanted Ti ions," IEEE Electron Device Lett., vol. 30, no. 12, pp. 1335-1337, Dec. 2009.
[7] M. Liu, Z. Abid, W. Wang, X. He, Q. Liu, and W. Guan, "Multilevel resistive switching with ionic and metallic filaments", Appl. Phys. Lett., vol. 94, p. 233106, Jun. 2009.

[8] X. Liu, Z. Ji, D. Tu, L. Shang, J. Liu, M. Liu, and C. Xie, "Organic nonpolar nonvolatile resistive switching in poly (3,4-ethylene-dioxythiophene): Polystyrenesulfonate thin film,' Organic Electronics, vol. 10, no. 6, pp. 1191-1194, Jun. 2009.

[9] S. Zhang, S. Long, W. Guan, Q. Liu, Q. Wang, and M. Liu, "Resistive switching characteristics of MnOx-based ReRAM," J. Phys. D: Appl. Phys., vol. 42, pp. 055112, Mar. 2009.

[10] Y. Wang, Q. Liu, S. Long, W. Wang, Q. Wang, M. Zhang, S. Zhang, Y. $\mathrm{Li}, \mathrm{Q}$. Zuo, J. Yang, and M. Liu, "Investigation of resistive switching in $\mathrm{Cu}$-doped $\mathrm{HfO}_{2}$ thin film for multilevel non-volatile memory applications," Nanotechnology, vol. 21, p. 45202, Jan. 2010.

[11] C. H. Chen, T. C. Chang, I. H. Liao, P. B. Xi, C. T. Tsai, P. Y. Yang, J. Hsieh, J. Chen, U. S. Chen, and J. R. Chen, "Tungsten nanocrystal memory devices improved by supercritical fluid treatment," Appl. Phys. Lett., vol. 91, no. 23, p. 232104, Dec. 2007.

[12] C. T. Tsai, P. T. Liu, T. C. Chang, C. W. Wang, P. Y. Yang, and F. S Yeh, "Low-temperature passivation of amorphous-silicon thin-film transistors with supercritical fluids," IEEE Electron Device Lett., vol. 28, no. 7 , pp. 584-586, Jul. 2007

[13] C. T. Tsai, T. C. Chang, P. T. Liu, P. Y. Yang, Y. C. Kuo, K. T. Kin, P L. Chang, and F. S. Huang, "Low-temperature method for enhancing sputter-deposited $\mathrm{HfO}_{2}$ films with complete oxidization," Appl. Phys. Lett., vol. 91, no 1, p. 12109, Jul. 2007.

[14] C. T. Tsai, T. C. Chang, K. T. Kin, P. T. Liu, P. Y. Yang, C. F. Weng, and F. S. Huang, "A low temperature fabrication of $\mathrm{HfO}_{2}$ films with supercritical $\mathrm{CO}_{2}$ fluid treatment," J. Appl. Phys., vol. 103, no. 7, p. 74108, Apr. 2008.

[15] M. C. Chen, T. C. Chang, S. Y. Huang, K. C. Chang, H. W. Li, S. C. Chen, J. Lu, and Y. Shi, "A low-temperature method for improving the performance of sputter-deposited $\mathrm{ZnO}$ thin-film-transistors with supercritical fluid," Appl. Phys. Lett., vol. 94, p. 162111, Apr. 2009.

[16] C. H. Chen, T. C. Chang, I. H. Liao, P. B. Xi, C. T. Tsai, P. Y. Yang, J. Hsieh, J. Chen, U. S. Chen, and J. R. Chen, "Tungsten nanocrystal memory devices improved by supercritical fluid treatment," Appl. Phys. Lett., vol. 91, no. 23, p. 232104, Dec. 2007.

[17] C. T. Tsai, P. T. Liu, T. C. Chang, C. W. Wang, P. Y. Yang, and F. S. Yeh, "Low-temperature passivation of amorphous-silicon thin-film transistors with supercritical fluids," IEEE Electron Device Lett., vol. 28, no. 7, pp. 584-586, Jul. 2007.

[18] P. T. Liu, C. T. Tsai, T. C. Chang, K. T. Kin, P. L. Chang, C. M. Chen, and Y. C. Chen, "Effects of supercritical fluids activation on carbon nanotube field emitters," IEEE Trans Nanotechnol, vol. 6, no. 1, pp. 29-34, Jan. 2007.

[19] K. H. Chen, Y. C. Chen, Z. S. Chen, C. F. Yang, and T. C. Chang, "Temperature and frequency dependence of the ferroelectric characteristics of $\mathrm{BaTiO}_{3}$ thin films for nonvolatile memory applications," Appl. Phys. A-Mater. Sci. Process, vol. 89, no. 2, pp. 533-536, Apr. 2007.

[20] C. T. Tsai, T. C. Chang, P. T. Liu, Y. L. Cheng, and F. S. Huang, "Low temperature improvement on silicon oxide grown by electron-gun evaporation for resistance memory applications," Appl. Phys. Lett., vol. 93, no. 5, p. 52903, Aug. 2008.

[21] C. T. Tsai, T. C. Chang, P. T. Liu, Y. L. Cheng, K. T. Kin, and F. S Huang, "Application of supercritical $\mathrm{CO}_{2}$ Fluid for dielectric improvement of SiOx film," Electrochem Solid State Lett., vol. 12, no. 2, pp. 35-37, Apr. 2009.

[22] S. C. Chen, T. C. Chang, H. H. Su, P.C. Yang, J. Lu, H. C. Huang, D. S. Gan, N. J. Ho, and Y. Shi, "Low-temperature ozone passivation for improving the quality of sputtered HfOx thin-film," Material Lett., vol. 63, no. 22, pp. 1914-1916, Sep. 2009

[23] M. C. Chen, T. C. Chang, S. Y. Huang, K. C. Chang, H. C. Huang, S. C. Chen, J. Liu, D. S. Gan, N. J. Ho, T. F. Young, G. W. Jhang, and Y. H. Tai, "Improvement of the performance of ZnO TFTs by low-temperature supercritical fluid technology treatment," Surface and Coatings Technology, vol. 204, pp. 1112-1115, Dec. 2009.

[24] K. H. Chen, T. C. Chang, G. C. Chang, Y. E. Hsu, Y. C. Chen, and H. Q. $\mathrm{Xu}$, "Low temperature improvement method on characteristics of $\mathrm{Ba}\left(\mathrm{Zr}_{0.1} \mathrm{Ti}_{0.9}\right) \mathrm{O}_{3}$ thin films deposited on indium tin oxide/glass substrates," Appl. Phys. A-Mater. Sci. Process, vol. 99, no. 1, pp. 291-295, Apr. 2010.

[25] K. C. Chang, T. C. Chang, T. M. Tsai, Y. E. Syu, H. C. Huang, Y. C. Hung, T. F. Young, D. S. Gan, and N. J. Ho, "Low-temperature synthesis of $\mathrm{ZnO}$ nanotubes by supercritical $\mathrm{CO}_{2}$ fluid treatment," Electrochem Solid State Lett., vol. 14, no. 9, pp. 47-50, Jul. 2011. 
Kai-Huang Chen was born in Kaohsiung City, Taiwan, R.O.C. on October 29, 1973. He received the B.S. and M.S. degrees in electron engineering from National Yunlin University of Science and Technology in 1999 and 2001, respectively. Besides, $\mathrm{He}$ received the electrical engineering of $\mathrm{Ph} . \mathrm{D}$. degrees at National Sun Yat-Sen University in 2007. Currently, he is an assistant professor at Department of electronic engineering and computer science of Tung Fang Design Institute. His current research interests are in the areas of NvFRAM devices, high dielectric thin film, and capacitor devices.

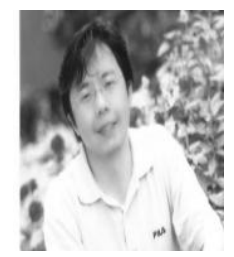

Sean Wu was born in Kaohsiung, Taiwan, on December 30, 1968. He received the B. S. degree in electrical engineering in 1993 from Chung Yuan Christian University in Taiwan. He received the M.S. and Ph.D. degrees in electrical engineering from National Cheng Kung University in 1995 and 2001, respectively.
He joined the Department of Electronics Engineering and Computer Science, Tung Fang Design University and became a professor in 2010. He also serves as the Department Head of Electronics Engineering and Computer Science from 2006 to 2008. He was the dean of academic affairs of Tung Fang Design University from 2011 to 2013. His research interests are the fabrication of piezoelectric thin films, design of acoustic wave devices, and substrate materials for SAW and FBAR devices.

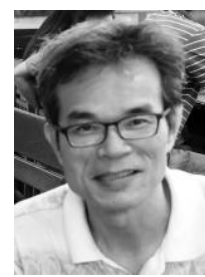

Chien-Min Cheng was born in Tainan City, Taiwan, R.O.C. on November 19, 1953. He received the B.S. and M.S. degrees in electron engineering from Chiao Tung University in 1989 and 1991, respectively. Besides, He received the electrical engineering of $\mathrm{Ph} . \mathrm{D}$. degrees at National Sun Yat-Sen University in 2008. Currently, he is a professor at Department of Electronic Engineering of Southern Taiwan University of Science and Technology. His current research interests are in the areas of piezoelectric devices and capacitor devices. 